Sci Transl Med. 2013 September 25; 5(204): 204ps13. doi:10.1126/scitranslmed.3007504.

\title{
Human Skin in the Game
}

\author{
Rachael A. Clark
}

${ }^{1}$ Department of Dermatology, Brigham and Women's Hospital, Harvard Medical School, Boston, MA 02115, USA

\section{Abstract}

Clinical and experimental observations in atopic dermatitis and psoriasis reveal new mechanisms of antiviral immunity and inflammation [Wolk et al., this issue; Kim et al., previous issue].

In addition to its function as a barrier tissue, human skin is a hotbed of immunological activity and plays host to a variety of resident and itinerant immune cells, including macrophages, dendritic cells, Langerhans cells and nearly 20 billion memory $\mathrm{T}$ cells $(1,2)$. Healthy human skin contains dendritic cells that can present antigens from foreign invaders and resident memory $\mathrm{T}$ cells that are poised to rapidly respond. Studies in mice have shown that pathogens can be recognized, presented, and defeated by skin-resident immune cells alone (3-5). Human epidermal keratinocytes also respond to infection by pumping out inflammatory cytokines, chemoattractants and a variety of antimicrobial and antiviral products (6).

The skin is an ideal tissue in which to study human immune responses in action. Skin immune reactions can be observed visually, easily biopsied, and when the pathologic process is understood, skin diseases can often be treated with topical medications. But despite its accessibility, our understanding of the biology underpinning many inflammatory skin diseases is poor and there are numerous clinical observations in dermatology that immunologists cannot yet explain. Two translational studies that advance our understanding of human skin diseases are summarized here $(7,8)$.

\section{Supercharged Viral Immunity in Psoriasis}

Careful examination of a clinical observation has led to an advance in our understanding of antiviral immunity in the skin. In this issue of Science Translational Medicine, Wolk and colleagues focused on the observation that patients with atopic dermatitis have increased incidences of both bacterial and viral infections, including eczema herpeticum, whereas patients with psoriasis rarely suffer from viral infections despite comparable skin-barrier breakdown. Bacterial susceptibility in atopic dermatitis is thought to result from reduced antibacterial protein (ABP) production caused by low levels of the ABP-inducing proinflammatory cytokine IL-17 (synthesized and secreted by Th17 cells) and high levels of the anti-inflammatory Th2 cytokine IL-10 but the differential susceptibility of atopic 
dermatitis and psoriasis patients to viral infections has never been explained (9-11). The authors found that the expression of antiviral proteins (AVP) was higher in psoriatic than atopic dermatitis skin lesions and that expression of AVP was localized to epidermal keratinocytes. Type-I interferons (IFN) are the best-known inducers of AVP but there was virtually no expression of type-I IFN in chronic psoriatic lesions, suggesting that another stimulus is responsible for increased AVP production. Transcriptional profiling of human skin samples demonstrated that AVP production correlated only with interleukin 29 (IL-29) mRNA levels. Moreover, IL-29 was present in the psoriatic skin lesions but absent from atopic dermatitis lesions and healthy skin.

IL-29 and the related cytokines IL-28A and IL-28B constitute a new subfamily of type-II IFNs (12). These cytokines signal through the IL-28 receptor 1 (IL-28R1)/IL-10R2 heterodimeric receptor, are produced at high concentrations by toll-like receptor (TLR)stimulated antigen-presenting cells (APCs) and at lower concentrations by other cell types, and have antiviral activity in vitro and in vivo (12-14). The authors found that IL-29 treatment of keratinocytes, three-dimensional epidermal cell cultures, and samples of healthy human skin up-regulated keratinocyte AVP production, an increase that was blocked by neutralizing IL-29 antibodies. In contrast to IL-22, IL-29 did not up-regulate ABP production, did not cause acanthosis (epidermal hyperplasia), and had no effect on the expression of molecules necessary for keratinocyte terminal differentiation.

The authors demonstrated by immunofluorescence analyses that IL-29 co-localized with T cells expressing the retinoic acid-related orphan receptor $\gamma \mathrm{t}(\mathrm{ROR} \gamma \mathrm{t})$, which implicates Th17 cells as major producers of IL-29 in psoriasis. Indeed, naïve human T cells differentiated in vitro toward the Th17 lineage produced more IL-29 than did APCs activated by TLR ligands. Specific inhibitors of ROR $\gamma$ t blocked IL-29 production and TGF $\beta$ was important for the generation of IL-29-producing Th17 cells. Inhibitors of both calcineurin-a protein phosphatase necessary for T cell activation-and c-Jun N-terminal kinases (JNKs) signaling proteins involved in $\mathrm{T}$ cell differentiation, proliferation and cell death-blocked IL-29 production, implicating these pathways in IL-29 synthesis by T cells. Lastly, keratinocytes treated in vitro with Th17 cell supernatants up-regulated production of both AVP and ABP but IL-29-blocking antibodies decreased only AVP production.

In addition to its clinical importance, this work contributes two additional findings to the field. First, it establishes that keratinocyte production of ABPs and AVPs is regulated in separate and different ways. ABP production can be induced by a number of mediators (Fig. 1), but AVP production is mediated only by IL-29 and, to a lesser extent, IFN $\gamma$. Second, this work implicates Th17 cells, and not APCs, as a major source of IL-29 in psoriatic skin lesions. This is an interesting example of the adaptive immune system triggering an immune response mediated by the innate immune system. It is well acknowledged that the innate immune system strongly influences adaptive immunity - for example, dendritic cells imprint $\mathrm{T}$ cells with specific functional activities and homing properties - but there has been less focus on the equally critical crosstalk from the adaptive to the innate immune system. Although often artificially divided, the adaptive and innate immune systems evolved together over the last 50 million years, and the function of each is intimately dependent on the other (15). 
Wolk et al. are to be commended for using human cells and tissues to decipher the mechanisms behind the differential viral susceptibilities in observed in atopic dermatitis and psoriasis patients. As a result, the findings are truly reflective of the human disease process and lessons learned are directly applicable to the treatment of human patients. A better understanding of how skin regulates AVP production could lead to improved therapies for viral infection of the skin as well as virally mediated cancers such as Merkel cell carcinoma, Kaposi's sarcoma and HPV-associated skin cancers.

\section{Inflammation's New Helper}

Innate lymphoid cells (ILC) are newly described innate immune cells that lack T cell antigen receptors, are derived from a common lymphoid progenitor, and produce many of the same cytokines as their adaptive helper T cell counterparts (16). The ILC family includes classical natural killer (NK) cells and three types of ILCs that are distinguished by their functional characteristics. Type 1 ILCs (ILC1s) produce interferon- $\gamma$ in a Tbet-dependent manner and functionally resemble T helper type 1 (Th1) cells. ILC2s produce the Th2 cytokines IL-5 and IL-13 and functionally resemble Th2 cells and ILC3s produce IL-17A, IL-22, or both and can functionally resemble Th17, Th22, or both cell types (17).

In mice, ILC2s regulate inflammation, influence the barrier function of epithelial cells, and mediate anti-helminth immunity in the lung and gut. These functions depend on epithelial cell production of IL-33 and IL-25 (18-21). In humans, ILC2s are enriched in human nasal polyps, and thymic stromal lymphopoietin (TSLP) produced by nasal epithelial cells enhances cytokine production by ILC2s in response to IL-33 secretion by epithelial cells.

In a recent article in Science Translational Medicine, Kim et al. identified a population of ILC2s that are present in healthy human skin and enriched in the skin lesions of atopic dermatitis (7). IL-33, IL-25, and TSLP are elevated in atopic dermatitis lesions, suggesting that these cytokines may activate ILC2s and induce production of type 2 cytokines. To investigate this hypothesis, the authors used a mouse model in which the vitamin D analog calcipotriol (MC 903) is topically applied to the skin, resulting in Th2 cytokine production and skin inflammation that phenotypically resembles atopic dermatitis. The authors found that ILC2s were present in the skin lesions of mice, that ILC2s isolated from the draining lymph nodes produced the Th2 cytokines IL-5 and IL-13 and that depletion of ILCs significantly decreased skin inflammation (Fig. 2). The adaptive immune system is thought to contribute heavily to the inflammation of atopic dermatitis, but the authors observed significant skin inflammation in T- and B-cell deficient Rag $^{-/-}$mice, suggesting that innate cells alone could mediate skin inflammation in this model. Moreover, this ILC-dependent inflammation did not require the presence of the canonical cytokines IL-33 or IL-25, but was instead critically dependent on the presence of TSLP produced by keratinocytes. Although ILC3s were observed in the skin lesions of these mice, inflammation was still present in RorC $^{-/-}$mice, which lack ILC3s, suggesting that ILC2s were responsible for the observed inflammation.

These studies are the first to report that ILC2 are increased in the skin lesions of human atopic dermatitis and that in mice, ILC2 can be major players in initiating skin 
inflammation. These findings are intriguing, but additional studies are needed before these results can be translated into improved patient care. In the skin lesions in people with atopic dermatitis, ILC2s comprised a very small fraction of the total immune cell population and their function and cytokine production profile was not evaluated. It is unclear whether ILC2s are recruited nonspecifically to the lesions as a result of inflammation or whether they play a causative inflammatory role. Nonetheless, these elegant and comprehensive studies demonstrate that in at least one mouse model, ILC2 can initiate skin inflammation.

\section{Homing in on Humans}

Mouse models have been critical to advancing our understanding of immunology. Most of the immunology-related Nobel Prizes in Physiology or Medicine awarded over the last 30 years recognize work performed in mouse models and the vast majority of manuscripts in higher impact journals focus exclusively on mouse studies. The ease of use, the ability to control experimental conditions, and the molecular clarity inherent in transgenic mouse model systems make it tempting to publish experiments on mice, revel in their intellectual clarity, and stop the line of investigation there, without demonstrating that similar findings are also present in humans.

However, $95 \%$ of the therapies that cure cancer in mouse models are not effective in human beings. There are critical immunological differences between mice and humans, and these differences are incompletely characterized, making it difficult to predict which findings in mouse disease models will also hold true in human patients. Observations in mice accumulate in the literature, like players piling up on third base, without the hitscorrelative human studies-needed to bring them home and establish whether or not they will be useful in the treatment of human diseases.

It is therefore commendable that the studies presented by Wolk et al. and Kim et al. were based on initial clinical or experimental dermatologic observations in humans. Such an approach guarantees from the outset that the question studied will be relevant to human biology. It is often the unexplained clinical observation or the unexpected experimental result that leads to fundamental scientific advances. By focusing experiments on the questions brought to us by our patients, we ensure the relevance of our findings to human disease. By closing the loop and determining whether observations made in animal models are applicable to human biology, we recognize and acknowledge that the highest calling we have as physicians and scientists is to improve the lives of our patients.

\section{References and Notes}

1. Clark RA, et al. The vast majority of CLA $+\mathrm{T}$ cells are resident in normal skin. The Journal of Immunology. Apr 1.2006 176:4431. [PubMed: 16547281]

2. Zaba LC, Fuentes-Duculan J, Steinman RM, Krueger JG, Lowes MA. Normal human dermis contains distinct populations of CD11c+BDCA-1+ dendritic cells and CD163+FXIIIA+ macrophages. The Journal of clinical investigation. Sep.2007 117:2517. [PubMed: 17786242]

3. Wakim LM, Waithman J, van Rooijen N, Heath WR, Carbone FR. Dendritic cell-induced memory T cell activation in nonlymphoid tissues. Science. Jan 11.2008 319:198. [PubMed: 18187654] 
4. Gebhardt T, et al. Memory T cells in nonlymphoid tissue that provide enhanced local immunity during infection with herpes simplex virus. Nature immunology. 2009; 10:524. [PubMed: 19305395]

5. Jiang X, et al. Skin infection generates non-migratory memory CD8+ TRM cells providing global skin immunity. Nature. Mar 8.2012 483:227. [PubMed: 22388819]

6. Kondo S. The Roles of Keratinocyte-Derived Cytokines in the Epidermis and Their Possible Responses to UVA-Irradiation. J Investig Dermatol. 1999; 4:177.

7. Kim BS, et al. TSLP elicits IL-33-independent innate lymphoid cell responses to promote skin inflammation. Sci Transl Med. Jan 30.2013 5:170ra16.

8. Wolk K, et al. IL-29 is produced by $\mathrm{T}(\mathrm{H}) 17$ cells and mediates the cutaneous antiviral competence in psoriasis. Sci Transl Med. Sep 25.2013 5:204ra129.

9. Wolk K, et al. Deficiency of IL-22 contributes to a chronic inflammatory disease: pathogenetic mechanisms in acne inversa. J Immunol. Jan 15.2011 186:1228. [PubMed: 21148041]

10. Harder J, et al. Enhanced expression and secretion of antimicrobial peptides in atopic dermatitis and after superficial skin injury. The Journal of investigative dermatology. May.2010 130:1355. [PubMed: 20107483]

11. Ong PY, et al. Endogenous antimicrobial peptides and skin infections in atopic dermatitis. The New England journal of medicine. Oct 10.2002 347:1151. [PubMed: 12374875]

12. Witte K, Witte E, Sabat R, Wolk K. IL-28A, IL-28B, and IL-29: promising cytokines with type I interferon-like properties. Cytokine \& growth factor reviews. Aug.2010 21:237. [PubMed: 20655797]

13. Kotenko SV, et al. IFN-[lambda]s mediate antiviral protection through a distinct class II cytokine receptor complex. Nature immunology. 2003; 4:69. [PubMed: 12483210]

14. Sheppard P, et al. IL-28, IL-29 and their class II cytokine receptor IL-28R. Nature immunology. 2003; 4:63. [PubMed: 12469119]

15. Laird DJ, De Tomaso AW, Cooper MD, Weissman IL. 50 million years of chordate evolution: seeking the origins of adaptive immunity. Proceedings of the National Academy of Sciences of the United States of America. Jun 20.2000 97:6924. [PubMed: 10860947]

16. Spits H, Cupedo T. Innate lymphoid cells: emerging insights in development, lineage relationships, and function. Annual review of immunology. 2012; 30:647.

17. Spits $\mathrm{H}$, et al. Innate lymphoid cells--a proposal for uniform nomenclature. Nature reviews. Feb. 2013 13:145.

18. Monticelli LA, et al. Innate lymphoid cells promote lung-tissue homeostasis after infection with influenza virus. Nature immunology. Nov.2011 12:1045. [PubMed: 21946417]

19. Price AE, et al. Systemically dispersed innate IL-13-expressing cells in type 2 immunity. Proceedings of the National Academy of Sciences of the United States of America. Jun 22.2010 107:11489. [PubMed: 20534524]

20. Neill DR, et al. Nuocytes represent a new innate effector leukocyte that mediates type-2 immunity. Nature. 2010; 464:1367. [PubMed: 20200518]

21. Chang YJ, et al. Innate lymphoid cells mediate influenza-induced airway hyper-reactivity independently of adaptive immunity. Nature immunology. Jul.2011 12:631. [PubMed: 21623379] 


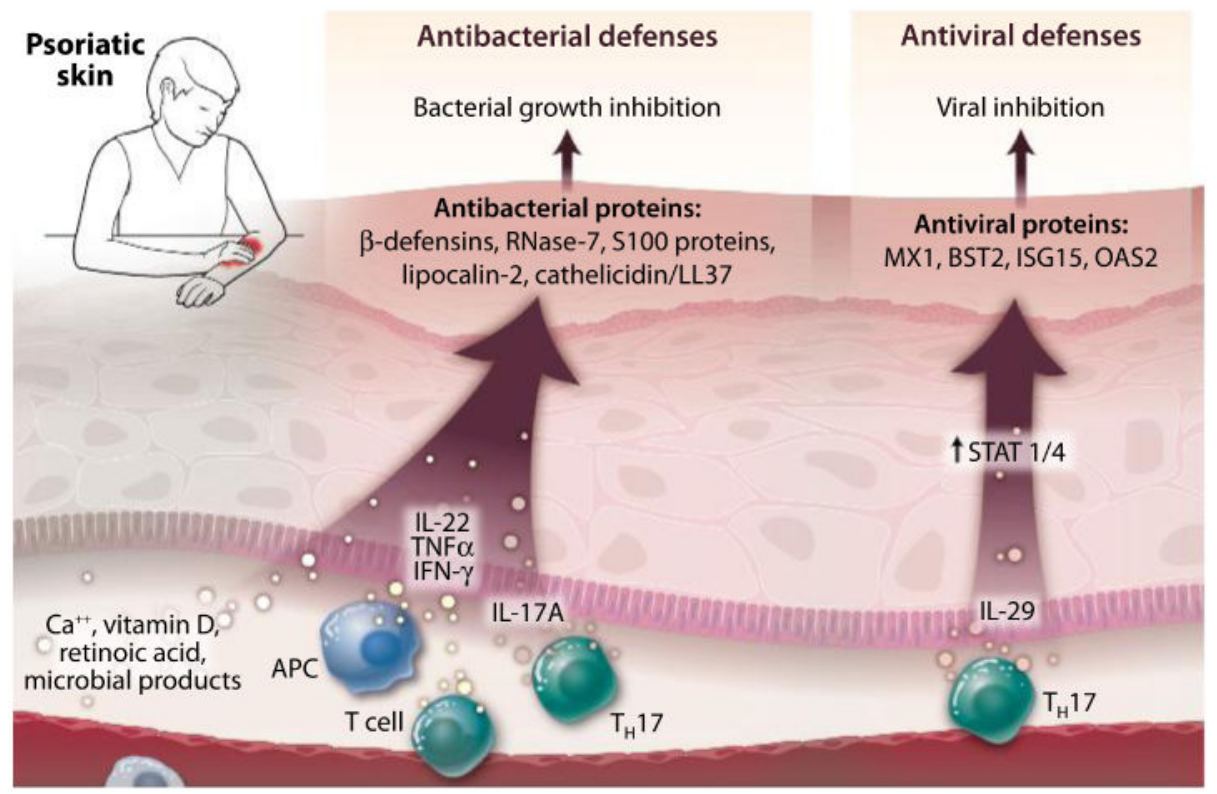

Figure 1. The production of antibacterial and antiviral proteins in psoriatic skin are regulated by different mechanisms

Antibacterial proteins can be induced by a variety of stimuli, including calcium, vitamin D, retinoic acid, microbial products and cytokines derived from both T cells and APC. In contrast, the production of antiviral proteins was closely linked to the production of IL-29, and to a lesser extent IFN $\gamma$, and the major source of IL-29 production was found to be Th17 cells resident within psoriatic skin lesions. 


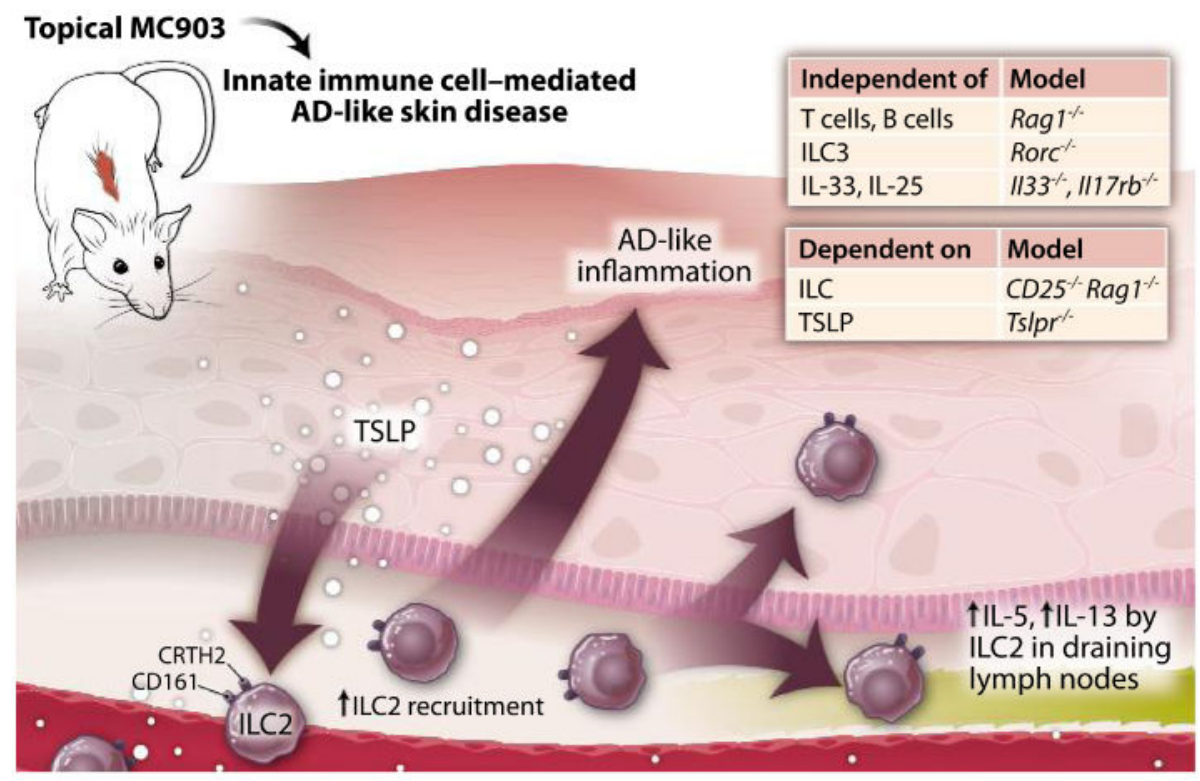

Figure 2. ILC2 initiate skin inflammation independent of the adaptive immune system in a mouse model of atopic dermatitis (AD)

Topical application of the vitamin D analog MC903 induces skin inflammation with histologic features reminiscent of human AD. ILC2s were observed in the skin and draining lymph nodes of treated mice and were found to mediate skin inflammation that was independent of T and B cells, ILCs and the canonical ILC2 cytokines IL-33 and IL-25, but was critically dependent on keratinocyte production of TSLP. 